Annuaire suisse de politique de développement

27-2 | 2008

Migration et développement: un mariage arrangé

\title{
L'aide au retour de la Suisse : bilan et perspectives
}

\author{
Eric Kaser et Saskia Schenker
}

\section{CpenEdition}

\section{Journals}

Édition électronique

URL : http://journals.openedition.org/aspd/644

DOI : 10.4000/aspd.644

ISSN : 1663-9669

\section{Éditeur}

Institut de hautes études internationales et du développement

\section{Édition imprimée}

Date de publication : 1 décembre 2008

Pagination : 199-212

ISBN : 978-2-940415-07-6

ISSN : 1660-5934

\section{Référence électronique}

Eric Kaser et Saskia Schenker, «L'aide au retour de la Suisse : bilan et perspectives », Annuaire suisse de politique de développement [En ligne], 27-2 | 2008, mis en ligne le 01 octobre 2010, consulté le 08 septembre 2020. URL : http://journals.openedition.org/aspd/644; DOI : https://doi.org/10.4000/aspd. 644 


\title{
L'aide au retour de la Suisse: bilan et perspectives
}

\author{
Eric Kaser et Saskia Schenker*
}

\section{Introduction}

Le système d'aide au retour mis en place par la Suisse a fêté son dixième anniversaire en 2007. L' instrument de l'aide au retour a donné d'emblée de bons résultats, ce qui a conduit à l'étoffer de plus en plus. Le présent article a pour but de décrire l'évolution de l'aide au retour depuis son instauration jusqu'en 2008 ; il en présente les diverses facettes et dresse un bilan de ces dix ans d'activité, et s'achève par un aperçu des perspectives d'avenir dans ce domaine.

Qui, en Suisse, est susceptible de bénéficier aujourd'hui d'une aide au retour dans son pays? Toutes les personnes relevant du domaine de l'asile peuvent déposer une demande d'aide au retour auprès des services-conseils en vue du retour gérés par les cantons, les centres d'enregistrement ou les aéroports de transit. Les réfugiés reconnus désireux de rentrer dans leur pays d'origine peuvent également bénéficier de l'aide au retour. Sont par contre exclus de l'aide au retour les délinquants et les personnes dont le comportement a été abusif pendant ou après la procédure.

Certains groupes de personnes relevant du domaine des étrangers ont également accès à l'aide au retour: victimes ou témoins de la traite des êtres humains, ainsi qu'artistes de cabaret séjournant en Suisse en situation d'exploitation.

Les catégories de personnes suivantes du domaine de l'asile ont accès aux conseils en vue du retour: les requérants d'asile dont la procédure est en cours, les requérants d'asile déboutés, les personnes frappées d'une décision de nonentrée en matière, les personnes admises à titre provisoire, les personnes à protéger et les refugiés reconnus ${ }^{1}$.

\section{Aperçu historique de l'aide au retour suisse}

\section{Premières mesures d'aide au retour}

C'est en 1959 déjà que la Division de police du Département fédéral de justice et police (DFJP) a élaboré un premier dossier sur le retour des réfugiés dans leur pays d'origine. Jusqu'au milieu des années 1980, la Confédération payait au cas

* Eric Kaser, chef de la section Aide au retour, Office fédéral des migrations (ODM), Département fédéral de justice et police (DFJP).

Saskia Schenker, collaboratrice à la section Aide au retour, Office fédéral des migrations (ODM), Département fédéral de justice et police (DFJP).

1 ODM, «Aide au retour», <http://www.bfm.admin.ch/bfm/fr/home/themen/rueckkehr/rueckkehrfoerderung. html>. 
par cas le billet d'avion ou les frais de transport de réfugiés sud-américains nécessiteux, ou leur versait de l'argent pour le voyage. Le principe de l'aide au retour actuelle remonte à l'année 1985. Confrontée à des demandes dans ce sens de certaines œuvres d'entraide lors de cette période caractérisée par un reflux de réfugiés sud-américains et par une révision imminente de la Loi sur l'asile, la Division Réfugiés de l'Office fédéral de la police a alors proposé de créer un groupe de travail interdépartemental d'aide au retour (voir l'encadré sur la chronologie des mesures d'aide au retour).

\section{Chronologie des mesures d'aide au retour}

- 1959: élaboration par la Division de police du Département fédéral de justice et police (DFJP) d'un premier dossier sur le retour des réfugiés dans leur pays d'origine, à la suite d'une demande d'assistance pour l'obtention de documents de voyage pour deux réfugiés hongrois désireux de rentrer chez eux.

- Du milieu des années 1970 jusqu'au milieu des années 1980: prise en charge du billet d'avion ou des frais de transport et versement d'une somme pour le voyage, en réponse à des demandes individuelles déposées en majeure partie par des réfugiés sud-américains.

- 1985: premier groupe de travail interdépartemental traitant de l'aide au retour (Division Réfugiés de I'Office fédéral de la police et DFAE).

- 1986: groupes de travail interdépartementaux Chili, Zaïre (aujourd’hui République démocratique du Congo) et Turquie; la loi révisée sur l'asile contient une disposition sur l'octroi de l'aide au retour.

- 1987: lancement du premier programme d'aide au retour (pour l'aide au retour et la réintégration professionnelle de ressortissants chiliens); premiers services-conseils en Suisse. Au Chili, ce programme était mis en œuvre par I'Intergovernmental Committee for Migration (Comité intergouvernemental pour les migrations, CIM), ancienne appellation de l'actuelle Organisation internationale pour les migrations (OIM).

- 1993: création du premier projet suisse d'aide au retour par l'Office fédéral des réfugiés (ODR) et désignation par l'ODR d'un chef de projet d'aide au retour.

๑ 1994: ouverture du Bureau de l'OIM à Berne.

ـ 1996 : lancement du programme pilote pour la Bosnie-et-Herzégovine.

ـ 15 janvier 1997: institutionnalisation de l'aide au retour pour l'ensemble des requérants d'asile, par I'envoi de directives concernant, d'une part, les conseils en vue du retour et, d'autre part, l'aide au retour individuelle; développement du réseau de conseil en vue du retour; lancement du programme d'aide au retour en Bosnie-et-Herzégovine (1997-1999).

- 1998: adoption de dispositions sur l'aide au retour dans la Loi sur l'asile.

- 1999: création de la section Aide au retour dans I'ODR; programme d'aide au retour pour le Kosovo (1999-2001).

\section{Développement de programmes spécifiques à certains pays}

Le programme d'aide au retour en Bosnie-et-Herzégovine a été le premier du genre à concrétiser le principe d'un encouragement systématique du retour volontaire à l'échelle de la Confédération. Il a permis, en l'espace de deux ans et demi, à quelque 10000 personnes de rentrer chez elles de leur plein gré. Les expériences faites à cet égard ont conduit à compléter les structures de l'Office fédéral des réfugiés (ODR) en créant une section Aide au retour, tandis que l'on étoffait simultanément le réseau - toujours existant - des services-conseils en vue du retour mis en place dans les cantons. C'était ainsi l'adoption définitive 
de l'idée selon laquelle des incitations appropriées, des informations ciblées et des conseils judicieux pouvaient encourager des réfugiés et des requérants d'asile à rentrer dans leur pays.

Après la fin des hostilités au Kosovo et avec la présence de troupes de maintien de la paix dans la région, un arrêté du Conseil fédéral datant du 16 août 1999 a levé l'admission provisoire collective des personnes déplacées par la guerre, et fixé un délai pour leur départ. L'ODR s'est alors associé à la Direction du développement et de la coopération (DDC), du Département fédéral des affaires étrangères (DFAE), et à l'Organisation internationale pour les migrations (OIM) afin de mettre sur pied le programme suisse d'aide au retour Kosovo. Doté d'un budget de 184 millions de francs, ce programme a pris une ampleur sans précédent: outre les prestations d'aide au retour proprement dite, il comprenait une participation à la reconstruction du Kosovo par la communauté internationale démarche profitant à l'ensemble de la population kosovare et non seulement aux rapatriés. C'est la DDC qui a pris en charge l'étude et la mise en œuvre de ces mesures d'aide structurelle.

Bien que ces deux programmes d'aide au retour dans les Balkans occidentaux aient revêtu un caractère exceptionnel tant par le nombre de personnes rapatriées que par le coût global des opérations qu'ils impliquaient, leurs résultats ont été si probants que ces expériences ont pu être mises à profit pour les programmes suivants - même si leur envergure était plus modeste.

Les programmes par pays sont élaborés par la section Aide au retour de l'actuel Office fédéral des migrations (ODM), en collaboration avec la DDC et l'OIM Berne. Il s'agit de prendre des mesures bien adaptées aux catégories de personnes à rapatrier et à la situation qui règne dans leur pays d'origine. Ces programmes spécifiques ont un volet «aide au retour» incluant le financement d'un partenaire sur place, et un volet «aide structurelle» assumé par la DDC. La composante «aide au retour» comporte des prestations individuelles qui vont au-delà du retour proprement dit. Les programmes réalisés après les premiers consacrés aux Balkans sont énumérés dans l'encadré suivant.

\section{Programmes par pays}

ـ 2000 : programme Sri Lanka (2000-2004).

- 2001 : programmes Ethiopie (2001-2002), République fédérale de Yougoslavie (2001-2003), nord de I'Irak (2001-2003), Somalie (2001-2002).

- 2002: programmes Angola (2002-2007), Iran (2002-2003), Macédoine (2002-2003), minorités du Kosovo (2002-2003)

ـ 2003: programmes Balkans pour les personnes vulnérables (2003-2006), Irak (depuis 2003), Turquie (2003-2005).

ـ 2004 : programmes Arménie (2004-2008), République démocratique du Congo (2004-2006).

๑ 2005: programmes Nigeria (depuis 2005), Afrique occidentale (depuis 2005).

- 2006: programmes Ethiopie (2006-2008), Afghanistan (2006-2008), Géorgie (depuis 2006), Maghreb (depuis 2006)

- 2007: contribution du Groupe directeur interdépartemental d'aide au retour (ILR) aux partenariats migratoires des Balkans occidentaux et au programme d'aide au retour des personnes vulnérables et des minorités du Kosovo (2007-2009). 
L'article 93, "Aide au retour et prévention de la migration irrégulière», de la Loi sur l'asile constitue la base légale de cette aide en Suisse. Les articles 62 à 78 de l'Ordonnance 2 sur l'asile décrivent le but de l'aide au retour, les bénéficiaires et les motifs d'exclusion.

Nous présentons ci-après les instruments suivants de l'aide suisse au retour: l'aide au retour individuelle, les services-conseils en vue du retour, l'aide au retour à partir des centres d'enregistrement et de procédure, le projet-pilote LEtr, les projets d'aide structurelle et la prévention de la migration irrégulière.

\section{Aide au retour individuelle}

Parallèlement aux programmes spécifiques à certains pays, l'aide au retour individuelle fait partie des prestations de base applicables pour tous les pays d'origine. Les prestations de cette aide individuelle se sont étoffées ces dernières années, et leur volume est aujourd'hui du même ordre que celui de l'aide au retour accordée dans le cadre d'un programme spécifique. Quel que soit son pays d'origine, toute personne relevant du domaine de l'asile et désireuse de rentrer chez elle a droit aux prestations gratuites du service de consultation du canton où elle séjourne, à l'organisation de son retour et au paiement de ses frais de voyage. Si le séjour en Suisse a dépassé trois mois, l'aide au retour comprend en outre une aide financière de départ de 1000 francs par adulte et de 500 francs par mineur. L'aide à la réintégration (aide complémentaire matérielle) s'élève à 3000 francs au maximum et peut être accordée pour différents motifs: profession et formation, logement, transport de matériel ou accueil par la famille (cash for shelter, cash for care). De l'aide peut en outre être octroyée sur présentation d'un certificat médical, pour la poursuite du traitement après le retour.

Le versement de l'aide au retour, le soutien apporté dans le cadre d'un projet professionnel et les recherches à effectuer en matière de structures sociales ou médicales s'effectuent en faisant appel à des partenaires locaux - missions de l'OIM, représentations diplomatiques de la Suisse ou organisations non gouvernementales (ONG).

Mais l'aide au retour a pour seul but de faciliter celui-ci et d'apporter une aide initiale à la réintégration dans le pays d'origine. L'accompagnement assuré par l'OIM dure en règle générale entre six mois et un an. Il n'appartient pas à ce système d'aide de fournir une assistance de longue durée ou une garantie de réinsertion réussie.

Depuis l'entrée en vigueur en 2008 de la nouvelle Loi sur l'asile, l'aide au retour ne peut être refusée que si la personne censée en bénéficier (Ordonnance 2 sur l'asile, art. 64) a commis des délits, contrevient à l'obligation de collaborer ou dispose de moyens financiers suffisants. Par ailleurs, l'usage pratiqué depuis mai 2007 de ne pas octroyer d'aide au retour individuelle à des personnes poursuivant leur route vers un Etat de l'Union européenne (UE) ou de l'Association européenne de libre-échange (AELE), ou vers un pays d'émigration traditionnel (Etats-Unis, Canada ou Australie), a été légalisé (Ordonnance 2 sur l'asile, art. 76). On ne peut bénéficier qu'une seule fois de l'aide au retour: 
si une personne rentrée librement dans son pays dépose une nouvelle demande d'asile en Suisse, elle est tenue de restituer l'aide qu'elle avait obtenue.

\section{Graphique 1: Répartition des besoins}

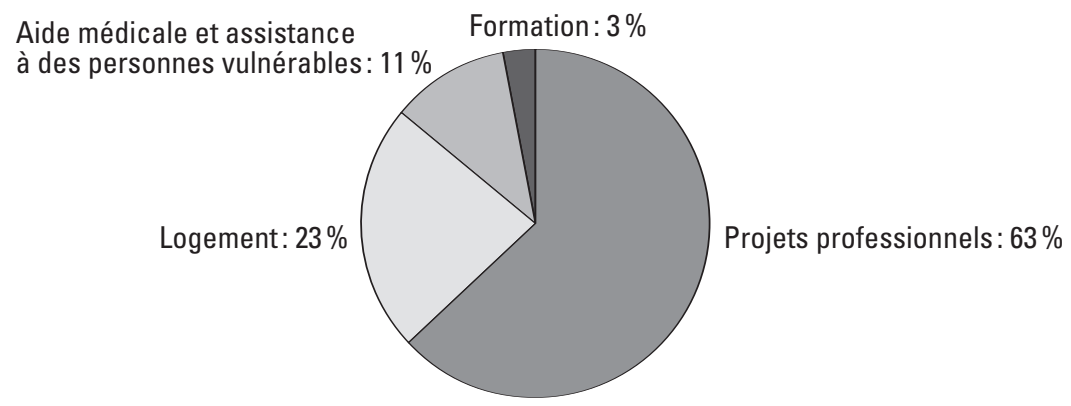

Source: Section Aide au retour, ODM, 2007.

\section{Services-conseils en vue du retour (CVR)}

Les services-conseils en vue du retour (CVR) sont mis en place et gérés par les cantons et financés par l'Office fédéral des migrations (ODM). Ils ont pour tâche d'aider les ayants droit à concrétiser leurs perspectives de retour. Leurs activités consistent notamment à informer les personnes susceptibles de rentrer volontairement dans leur pays d'origine, à maintenir un dialogue sur ce sujet avec le groupe cible en question et à faire le lien avec les autorités cantonales et communales. Le CVR s'occupe concrètement de chaque cas particulier (case management), depuis la première consultation jusqu'au départ de la personne considérée. Il assure la coordination avec la section Aide au retour de l'ODM et avec l'OIM Berne.

\section{Aide au retour à partir des centres d'enregistrement et de procédure (REZ)}

C'est en 2005 qu'a été instauré le projet «Aide au retour à partir des centres d'enregistrement et de procédure» (REZ). Dès leur entrée en Suisse, les personnes qui arrivent dans l'un des quatre centres d'enregistrement et de procédure, ou dans le centre de transit de l'ODM, se voient octroyer une aide au retour limitée à des conseils et à un forfait de 500 francs. Ce projet est mis en œuvre par le Bureau de l'OIM Berne.

\section{Projet pilote LEtr}

L'article 60 de la Loi fédérale sur les étrangers (LEtr), entrée en vigueur le $1^{\text {er }}$ janvier 2008, permet désormais de soutenir le départ volontaire et dans les délais prescrits de personnes particulièrement vulnérables. Le projet pilote LEtr offre ainsi à certaines catégories de personnes relevant du domaine des étrangers la possibilité de bénéficier d'une aide au retour. Prévu pour deux ans, il concerne d'une part les victimes et les témoins de la traite des êtres humains et, d'autre part, les personnes particulièrement menacées d'être exploitées dans 
l'exercice de leur activité lucrative. Il s'agit là du premier projet «thématique», donc sans lien direct avec le pays d'origine des bénéficiaires.

\section{Aide structurelle et prévention de la migration irrégulière (PIM)}

L'aide structurelle est possible dans tout pays dont des ressortissants bénéficient d'une aide au retour octroyée par l'ODM; elle poursuit les objectifs suivants :

$\checkmark$ réduire les disparités entre retournants et personnes restées sur place, en permettant également à ces dernières de bénéficier de projets d'aide structurelle;

$\checkmark$ inciter les Etats de provenance à réadmettre leurs ressortissants et à s'engager à mettre en œuvre des mesures de réintégration;

$\checkmark$ développer les compétences des pays de provenance en matière de gestion de la migration;

๑ participer au développement de relations de partenariat migratoire et encourager le dialogue migratoire.

L'Ordonnance 2 sur l'asile relative au financement spécifie (art. 72, al. 2) qu'il appartient à la DDC de mettre en œuvre les programmes à l'étranger de l'ODM. Cette aide structurelle, financée par le budget d'aide au retour de l'ODM, fait figure de mandat supplémentaire pour la DDC. Outre ses propres programmes découlant de la Loi fédérale sur la coopération au développement et l'aide humanitaire internationales, la DDC assume diverses tâches de coopération avec des partenaires extérieurs. Ces «activités tierces» sont conformes aux principes fondamentaux de la DDC, mais avec des priorités différentes. Les projets d'aide structurelle financés par l'ODM sont ainsi examinés et mis au concours en tenant compte des critères habituels de la DDC en la matière. L'ODM recourt aux compétences de la DDC en évitant d'instrumentaliser les opérations de coopération au développement ou d'aide humanitaire pour ses propres intérêts.

En vertu du nouvel article 93 de la Loi sur l'asile en vigueur depuis le $1^{\text {er }}$ janvier 2008, les programmes à l'étranger peuvent également viser à prévenir la migration irrégulière (PIM). Le Groupe directeur interdépartemental d'aide au retour (ILR) a ainsi mis sur pied au début de l'année 2008 une équipe de projet «Prévention de la migration irrégulière». Ce groupe de travail mène dans les pays prioritaires de la Suisse des projets spécifiquement destinés à prévenir la migration irrégulière; après avoir reçu l'approbation de l'ILR, ces projets sont financés par l'ODM. Les projets réalisés dans le cadre du mandat du groupe de travail «Prévention de la migration irrégulière» ont pour objectif principal de contribuer rapidement, au niveau opérationnel, à réduire le phénomène de la migration irrégulière. Concrètement, ce mandat se traduit par des campagnes d'information et de sensibilisation destinées aux migrants potentiels et par une aide fournie aux migrants irréguliers lorsqu'ils rentrent chez eux à partir d'un pays de transit (migrants échoués ou stranded migrants). Les projets d'aide structurelle apportent par contre des solutions de rechange à la migration irrégulière. Les pays prioritaires en matière de PIM varient selon l'évolution de leur situation migratoire. 


\section{Groupe directeur interdépartemental d'aide au retour (ILR)}

Le Groupe directeur interdépartemental d'aide au retour (ILR) est l'organe chargé de statuer sur la réalisation de programmes spécifiques et de projets d'aide structurelle relevant de l'aide au retour. Il coordonne les actions entreprises dans ce domaine, examine les synergies potentielles des mesures prévues par la DDC et par la section Aide au retour de l'ODM ainsi que par d'autres instances, et harmonise ces mesures avec le comité du Groupe de travail interdépartemental pour les questions migratoires (comité IAM). L'ILR a été institutionnalisé en 1999, après une évaluation positive du travail que menaient conjointement l'ODR de l'époque, la DDC et l'OIM depuis 1996 dans le cadre du programme Bosnie-et-Herzégovine. L'ILR a pour conseillers permanents le directeur du Bureau de coordination de l'OIM Berne, la personne responsable du secteur Migration et Développement à la DDC ainsi qu'un représentant de la Direction politique (Division politique IV) du DFAE².

Cette institutionnalisation de l'ILR en vue d'une coordination supradépartementale dans le domaine de l'aide au retour constitue un bon moyen de renforcer les échanges d'informations et la cohérence du travail effectué dans ce domaine, et aussi d'exploiter des synergies possibles entre migration et développement.

\section{Contribution de I'ILR aux partenariats migratoires Suisse-Balkans occidentaux 2007-2009}

Une illustration du travail de coordination effectué par l'ILR est sa Contribution concernant les partenariats migratoires entre la Suisse et les Balkans occidentaux: Stratégie 2007-2009, Kosovo, Bosnie-et-Herzégovine, Serbie³.

Les quatre objectifs poursuivis par cette contribution de l'ILR sont ici les suivants:

$\checkmark$ contribuer au développement de partenariats migratoires entre la Suisse et la Bosnie-et-Herzégovine, la Serbie et le Kosovo;

$\checkmark$ encourager le retour volontaire des membres des minorités et des personnes vulnérables;

$\checkmark$ renforcer l'aptitude des Etats concernés à gérer la migration;

$\checkmark$ prévenir la migration irrégulière en soutenant le développement des structures sociales et économiques.

L'aspect «aide au retour» était axé sur la situation particulière des personnes vulnérables et des minorités du Kosovo. Il s'agissait de répondre aux besoins individuels des personnes qui rentraient dans leur pays, après avoir pris des renseignements sur place. On pouvait ainsi assurer une aide au retour efficace et complète, adaptée à chacun des retournants volontaires.

ILR, Mandat, Berne, ODM; DDC, 2005

3 ILR, Contribution de l'ILR concernant les partenariats migratoires entre la Suisse et les Balkans occidentaux: Stratégie 2007-2009, Kosovo, Bosnie-et-Herzégovine, Serbie, Berne, ODM; DDC, 2007. (Pour des détails sur les partenariats migratoires, voir la section qui leur est consacrée.) 
Concernant l'aide structurelle, trois grands axes thématiques ont été fixés :

$1^{\circ}$ réunir les capacités requises pour gérer la migration;

$2^{\circ}$ développer les aspects sociaux et économiques ;

$3^{\circ}$ offrir aux rapatriés une aide à la réintégration et des services d'information sur la migration.

Les priorités thématiques se déterminent dans le cadre du dialogue bilatéral avec le pays concerné, d'entente avec celui-ci. Il incombe à la DDC et à ses bureaux de coopération sur le terrain de concevoir des projets ad hoc et de préparer la demande à présenter à l'ILR, après que les projets en question ont été discutés au sein de l'équipe avec la section Aide au retour de l'ODM. La décision de financer les projets appartient à l'ILR, tandis que la DDC s'occupe de leur réalisation. Et c'est l'ODM qui gère le budget de 12 millions de francs consacré aux projets d'aide structurelle. Il s'y ajoute une participation de la Principauté du Liechtenstein à hauteur de 1,5 million de francs. Les projets d'ONG suisses dont l'objectif, les groupes cibles et la concentration thématique et géographique correspondent à la stratégie ILR «Partenariats migratoires Balkans occidentaux 2007-2009» (PMBO 07-09) peuvent bénéficier, dans le cadre de l'aide structurelle, d'une contribution financière limitée à $50 \%$ au maximum. Ces organisations d'entraide se voient ainsi associées au dialogue mené par l'ILR sur les partenariats migratoires Suisse-Balkans occidentaux.

\section{Bilan de dix ans d'aide au retour}

\section{Bilan de l'aide au retour proprement dite}

\section{$\square$ Adaptation à des circonstances en évolution}

Alors qu'elle se bornait dans les premiers temps au simple versement d'une somme forfaitaire, l'aide au retour a pris de l'ampleur avec des mesures de réintégration adaptées aux besoins personnels de chaque retournant volontaire. Cette évolution ne s'est pas limitée aux programmes spécifiques à des pays déterminés, car l'aide au retour individuelle offerte indépendamment du pays d'origine a également dû être adaptée à des situations qui évoluaient constamment. Après les retours en masse qui caractérisaient les premiers programmes pour les Balkans et avec les nombres désormais restreints de personnes partant avec une aide au retour, on constate une recrudescence des cas complexes et coûteux - notamment pour les personnes désireuses de rentrer dans leur patrie après un long séjour en Suisse, par exemple des malades, des femmes qui élèvent seules leurs enfants, des veuves, des retraités, des familles, des personnes souffrant de traumatismes de guerre ou qui ont eu des parcours spécialement longs et difficiles. Tous ces retournants volontaires ont besoin de renseignements approfondis et d'une assistance efficace avant de pouvoir entreprendre le retour dans leur pays d'origine. C'est pourquoi les prestations au titre de l'aide au retour individuelle atteignent largement le niveau de celles consacrées aux programmes spécifiques. Cela permet de mieux tenir compte du facteur de la motivation pour revenir au pays. Et l'on ne voit aujourd'hui plus de différences significatives entre l'élément «aide au retour» des programmes à l'étranger et le système d'aide au retour individuelle. 


\section{$\square$ Nombre des départs}

Depuis 1997, quelque 65000 personnes ont bénéficié de prestations d'aide au retour octroyées par la Suisse. On dénombre une moyenne de 1500 personnes par an pour ces cinq dernières années. Principale région concernée à l'origine, les Balkans restent une destination importante des retours volontaires. Le programme d'aide au retour dans les Balkans occidentaux compte toujours un nombre considérable de bénéficiaires; ce nombre atteint environ 50 personnes par année pour le programme incluant Bosnie-et-Herzégovine, Serbie, Kosovo et Monténégro, et une trentaine de personnes prenant le chemin de la Macédoine. Mais, ces dernières années, les retours volontaires prennent plus souvent la direction des pays de l'ex-Union soviétique et de l'Afrique. Le programme Géorgie enregistre, depuis son lancement en 2006, un peu plus de 30 retournants volontaires en moyenne par année. La Russie et l'Ukraine sont également parmi les pays où beaucoup de personnes reviennent avec une aide au retour individuelle. Quant à la Turquie, ce pays a vu revenir en 2006 et 2007 plus de 60 retournants volontaires venant de la Suisse. La majorité des retours à destination du continent africain s'inscrivent dans le cadre des programmes Nigeria, Afrique occidentale et Maghreb. Le programme Angola s'est achevé à la fin de 2007. Du côté de l'aide au retour individuelle, le Cameroun compte également un nombre important de retournants volontaires.

Certains événements politiques exercent aussi une influence sur les retours volontaires : au cours des années 2004 à 2006, plus de 110 personnes par an sont ainsi rentrées de leur plein gré en Irak dans le cadre du programme consacré à ce pays.

\section{$\square$ Evaluation, à titre d'exemple, du programme d'aide au retour pour les personnes vulnérables originaires des Balkans (2003-2006)}

Le programme d'aide au retour pour les personnes vulnérables originaires des Balkans (2003-2006) a été le premier à être axé sur les besoins individuels de ces personnes dont la situation ou l'état de santé posent des problèmes extraordinaires de réintégration dans leur pays d'origine ou rendent le retour difficile ou impossible à exiger, et qui constituent en même temps pour la Suisse des cas épineux en termes de renvoi ou d'intégration.

Sur les 252 personnes qui ont sollicité ce programme entre le $1^{\text {er }}$ juillet 2003 et le 31 décembre 2006, 205 sont rentrées dans leur pays d'origine, dont la moitié environ en Bosnie-et-Herzégovine. Il y a eu trois cas de «retour du retour».

Quant au profil familial, les familles et les personnes élevant seules leurs enfants formaient le plus grand groupe (124 personnes ou 59\% du total). Les célibataires représentaient $24 \%$ de l'ensemble; les couples, 16\%. Deux tiers des couples et une petite moitié des célibataires avaient plus de 60 ans. Cet intérêt marqué pour le retour - alors que la proportion des expatriés de plus de 60 ans est faible - s'explique le plus souvent par un manque d'intégration en Suisse ainsi que par le désir de passer le soir de leur vie dans leur patrie. Deux personnes rentrées avec une maladie incurable sont décédées peu après leur retour.

Près de trois quarts des participants à ce programme sont rentrés chez eux après avoir vécu longtemps ou très longtemps en Suisse: $42 \%$ étaient restés entre quatre et sept ans dans notre pays, et $31 \%$ y avaient séjourné plus de huit ans. 
Ils sont partis au moment où l'intégration devenait prioritaire, si ce n'était pas déjà chose faite.

Sur mandat du Groupe directeur interdépartemental d'aide au retour (ILR) et de l'équipe de projet Balkans, l'OIM Berne a dressé en automne 2006 un rapport de suivi concernant toutes les personnes rentrées dans leur pays entre le $1^{\text {er }}$ juillet 2003 et le 31 août 2006 dans le cadre du programme d'aide au retour pour les personnes vulnérables originaires des Balkans. Ce rapport avait pour but d'examiner si l'on avait pris des mesures de réintégration efficaces et durables.

Les résultats de cette étude sur des situations concrètes peuvent être qualifiés de positifs. Le fait que $90 \%$ environ des retournants volontaires habitent toujours le lieu où ils sont revenus indique que leur situation s'est stabilisée. On ne connaît que deux cas de personnes qui sont reparties ailleurs. Deux tiers de ces retournants ont signalé un motif d'insatisfaction - notamment l'absence d'un réseau familial digne de ce nom (par exemple à la suite d'un divorce, du décès de proches, de l'émigration d'une partie de la famille ou en raison de tensions internes), un mauvais état de santé ou le désir persistant d'émigrer en Europe occidentale. Cela montre que certaines améliorations structurelles restent parfois possibles, tout en sachant que des institutions extérieures ont une influence restreinte sur les circonstances de la vie privée. On a enregistré 37 cas de retournants obtenant de l'aide pour leur réintégration professionnelle, et dans 22 cas ces projets contribuent toujours au revenu des bénéficiaires en question, ce qui représente trois quarts de l'ensemble des projets d'aide au retour. L'intégration dans les structures du pays d'origine n'a posé aucun problème dans presque tous les cas.

Les prestations d'aide offertes dans le cadre de ce programme ont donné des résultats largement probants. En effet, l'essentiel des investissements effectués avec l'argent du programme reste exploité par les personnes retournées, de sorte que l'on peut parler d'un soutien produisant des effets durables ${ }^{4}$.

Dans son rapport final sur l'examen des démarches et des processus dans le domaine de la promotion du retour des requérants d'asile, le Contrôle fédéral des finances (CDF) a confirmé que les ressources étaient affectées aux programmes spécifiques à un pays de manière rationnelle, et estime que l'ILR, responsable en la matière, est «un exemple de collaboration interdépartementale pour les questions de politique intérieure comme de politique extérieure dans le domaine de la migration $»^{5}$.

\section{Bilan de l'aide structurelle basé sur l'exemple des Balkans}

Le programme d'aide au retour pour les personnes vulnérables originaires des Balkans (2003-2006) incluait une aide structurelle financée par l'ODM et fournie par la DDC, budgétée à 29 millions de francs. La réalisation des projets a été

4 Equipe de projet Balkans, ILR, Stratégie Balkans 2003-2006: Programme d'aide au retour dans les Balkans à l'intention des personnes vulnérables (Bosnie-et-Herzégovine, Serbie, Monténégro et

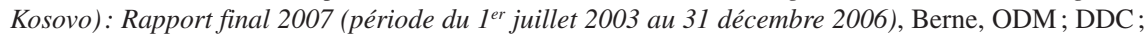
OIM, 2007.

5 Eidgenössische Finanzkontrolle (EFK), Bundesamt für Migration, Bereich Rückkehrförderung, EFK Bericht, nº 1.6196.420.00145.02, Bern, EFK, 20. Dezember 2006. 
menée par les services de l'aide humanitaire et de la coopération de la DDC, associés à divers partenaires - instances gouvernementales et ONG locales, organisations internationales (OIM, Haut-Commissariat des Nations unies pour les réfugiés [HCR]), ONG suisses. Au total, 49 projets ont ainsi obtenu l'approbation et le soutien de l'ILR.

Les projets réalisés dans le cadre de ce programme ont contribué de façon substantielle à stabiliser la situation sociale et économique des pays concernés. Parallèlement à leur aide individuelle, l'ODM et la DDC ont décidé en 2003 d'apporter ponctuellement des contributions financières à des projets d'aide aux petites structures, le but étant de renforcer les structures des lieux de retour et de faciliter la réintégration des retournants dans la société locale. Jusqu'à la fin de 2006, neuf projets financés par l'ODM ont ainsi été menés à bien par l'OIM sous la direction de la DDC. Les communes bénéficiaires ont quasiment toujours participé à ces projets, sous forme de contribution financière ou par des travaux d'installation. Cependant, l'évaluation globale des mesures d'aide aux petites structures a révélé un rapport peu satisfaisant entre travail et moyens financiers requis d'une part, durabilité de ces opérations ponctuelles d'autre part. On a ainsi renoncé à poursuivre l'aide aux petites structures dans le cadre de la stratégie de l'ILR 2007-2009 des partenariats migratoires avec les Balkans occidentaux.

Les liens établis au sein de l'équipe de projet Balkans, les échanges d'informations et le soutien apporté sur le terrain par les bureaux de coopération de la DDC peuvent servir de modèle pour la collaboration entre ODM et DDC en faveur d'autres régions du monde, dans le cadre de l'ILR. L'engagement accru de l'ODM dans des régions de l'ex-Union soviétique ou en Afrique renforcerait aussi la collaboration avec les divisions pour ces régions au sein de la DDC.

\section{Finances}

A la suite du recul du nombre des retournants volontaires après la période des grands programmes spécifiques aux Balkans, le budget de l'aide au retour a logiquement été revu à la baisse - et avec lui les dépenses effectives.

Les dépenses d'aide structurelle prélevées sur le crédit d'engagement ont également baissé régulièrement, pour se stabiliser actuellement à un niveau relativement bas. D'autres pays ou régions - pays de l'ex-Union soviétique, Afrique occidentale ou pays du Maghreb en tant que zones de transit - ont gagné en importance, conduisant à multiplier les projets d'aide structurelle et de prévention de la migration irrégulière dans ces régions (voir graphique 2). 


\section{Graphique 2: Dépenses d'aide au retour, 2000-2009}

En millions de francs

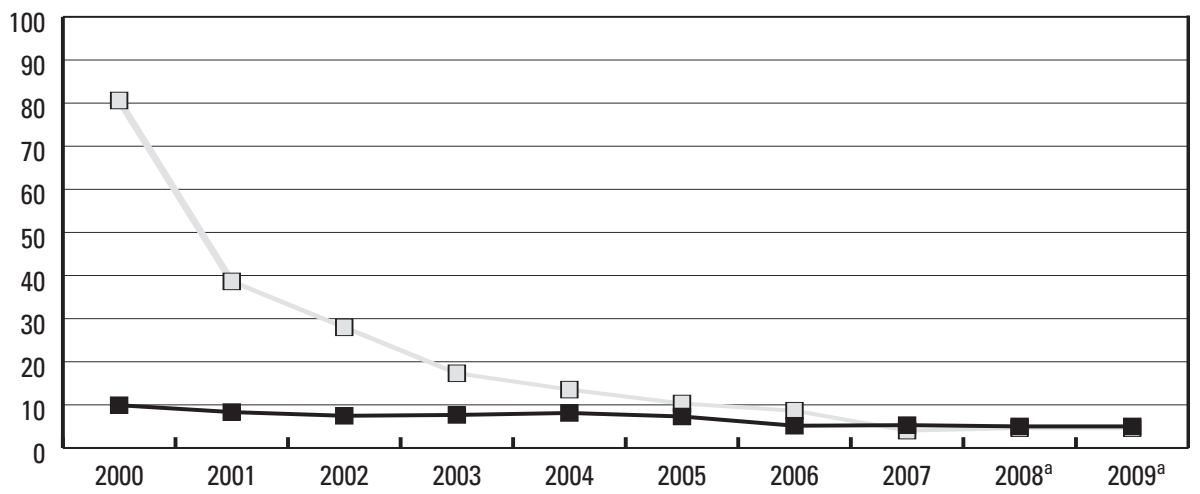

crédit d'aide général ${ }^{\mathrm{b}} \square$ crédit d'engagement $^{\mathrm{c}}, 235$ millions $^{2}$

\section{Données du graphique 2}

\begin{tabular}{lrrr}
\hline & Crédit d'aide général & Crédit d'engagement & Total \\
\hline 2000 & 9911832 & 80600000 & 90511832 \\
\hline 2001 & 8320528 & 38634339 & 46954867 \\
\hline 2002 & 7463968 & 27989425 & 35453393 \\
\hline 2003 & 7664740 & 17326355 & 24991095 \\
\hline 2004 & 8121000 & 13530994 & 21651994 \\
\hline 2006 & 7300000 & 10314450 & 17614450 \\
\hline 2007 & 5153838 & 8644634 & 13798472 \\
\hline $2008^{a}$ & 5290522 & 4027433 & 9317955 \\
\hline $2009^{a}$ & 5000000 & 4600000 & 9600000 \\
\hline
\end{tabular}

Source: Section Aide au retour de I'ODM, 2008.

a Les données pour 2008 et 2009 sont une prévision des dépenses.

b Crédit d'aide général = conseils en vue du retour et aide au retour individuelle.

c Crédit d'engagement = programmes spécifiques et aide structurelle.

\section{Perspectives d'avenir}

Le concept d'aide au retour englobe, sous le terme de «promotion de l'aide au retour», l'ensemble des mesures susceptibles d'encourager les requérants d'asile à rentrer volontairement dans leur pays d'origine. L'aide au retour présente des avantages par rapport au rapatriement sous contrainte, et c'est d'ailleurs la seule option possible lorsque celui-ci est inapplicable. L'aide au retour ainsi que les programmes spécifiques et les projets d'aide structurelle contribuent à rendre les autorités des pays d'origine mieux disposées à reprendre leurs ressortissants; ils sont un élément positif à faire valoir dans le dialogue migratoire en ce qu'ils facilitent la réintégration des personnes qui rentrent chez elles tout en contribuant à prévenir une nouvelle migration. L'aide au retour constitue ainsi un pilier important de la politique migratoire.

L'objectif premier et la tâche essentielle de l'aide au retour pratiquée par la Confédération est d'inciter les personnes relevant du domaine de l'asile et, en 
partie, de celui des étrangers à quitter la Suisse de leur plein gré ou de façon autonome. Un «départ volontaire», dans l'acception suisse, correspond à un retour dans le pays d'origine exempt d'obligation juridique, les étrangers en question ayant un statut qui leur donne le droit de séjourner en Suisse. Le départ est considéré comme «autonome» lorsque le droit de séjourner en Suisse expire et que l'étranger en question quitte le pays dans le délai prescrit et sans que l'on doive recourir à des mesures policières.

Après l'adaptation, ces dernières années, du volume et des sommes octroyées de l'aide au retour individuelle aux prestations des programmes spécifiques, des montants pour cette aide ont été fixés par voie d'ordonnance avec l'entrée en vigueur, au début de 2008, de la nouvelle Loi sur les étrangers et de la Loi sur l'asile modifiée. Cette solution s'est avérée judicieuse pour l'aide individuelle. Il apparaît en outre que des prestations accrues constitueraient un «facteur pull $\gg^{6}$ susceptible de rendre la Suisse excessivement attrayante. Des expériences de cet ordre ont été faites en 2007, lorsque la Suisse a reçu un grand nombre de demandes d'asile en provenance de Roumanie: ces personnes justifiaient entre autres leur requête par les problèmes économiques et sociaux qui rendaient la vie difficile dans leur pays, et affirmaient avoir entendu dire que la Suisse se caractérisait par d'excellentes conditions de vie et qu'elle accorde une aide au retour. Cela a conduit l'ODM à décider, le 7 mai 2007, que les ressortissants des pays de l'UE n'auraient plus droit à l'aide au retour. Il n'existe actuellement aucune raison concrète de penser que l'aide au retour soit un facteur d'attraction pour des ressortissants de certains pays. Les statistiques de l'asile permettraient à l'ODM de déceler très rapidement une tendance de ce genre et de prendre des mesures pour la désamorcer.

Le projet pilote LEtr permet de se faire une première idée des résultats possibles de l'aide au retour destinée aux personnes relevant du domaine des étrangers. Pour savoir s'il convient d'étendre cette aide à d'autres groupes cibles du même domaine, on se basera sur les expériences acquises grâce à ce projet, ainsi que sur d'autres éléments d'appréciation (évolution de la situation).

L'activité centrale de l'aide au retour - à savoir l'organisation de chaque retour volontaire et autonome en fonction des circonstances individuelles - reste essentielle. Par ailleurs, les grands programmes de longue durée consacrés à des pays déterminés, incluant aide au retour et aide structurelle, vont diminuant. Avec le développement des prestations inhérentes à l'aide au retour individuelle, l'application de programmes spécifiques ne se justifie plus guère que dans certains contextes particuliers. Ce pourrait être le cas lorsque la situation sur place ou un nombre considérable de retournants vulnérables demanderait une assistance accrue ou des prestations dépassant les moyens dont dispose l'aide au retour individuelle.

L'offre d'aide structurelle et de projets destinés à prévenir la migration irrégulière reste valable pour tous les pays dont les ressortissants bénéficient d'une aide au retour de l'ODM. Ces projets continueront à l'avenir de profiter en premier

6 Cette expression fait référence au «modèle push-pull» (répulsion-attraction) de la recherche scientifique en matière migratoire: les facteurs push sont les conditions du pays d'origine qui incitent à la migration (crise économique, persécution, guerre, etc.); les facteurs pull correspondent aux incitations du pays d'accueil (prospérité économique, stabilité politique, démocratie, etc.) (NDLR). 
lieu aux populations locales. Aide structurelle et projets PIM s'inscrivent dans une perspective qui associe le développement et les intérêts de l'ODM en matière de politique migratoire.

Le système helvétique d'aide au retour est bien étoffé par rapport à celui d'autres Etats d'Europe occidentale. On constate ainsi que les conditions de réintégration des retournants varient selon le pays européen où les retournants ont séjourné. Au sein de l'Union européenne, les échanges d'informations à ce sujet se font à intervalles irréguliers. Il n'y a ni analyses systématiques ni véritable coordination. Il serait souhaitable que l'on se renseigne réciproquement et que l'on harmonise les divers instruments et prestations d'aide au retour dans le cadre de la coopération entre Etats européens.

\section{Conclusion}

Le nombre de retournants volontaire n'est pas le seul critère de réussite d'un système d'aide au retour. Il s'agit de faire en sorte que les différents instruments utilisés contribuent à une stratégie sans lacunes, afin d'assurer le retour - volontaire, obligatoire ou sous contrainte - autant que possible en coopération avec le pays considéré. Le but, à moyen et à long terme, est de contribuer à une meilleure coopération avec les Etats d'origine en matière de politique migratoire et d'offrir une solution plus positive que le retour forcé.

Combiné aux instances partenaires réunies dans le Groupe directeur interdépartemental d'aide au retour (ILR), le système d'aide au retour de la Confédération apporte des contributions ciblées aux objectifs suisses en matière de politique étrangère et de politique migratoire - comme cela s'est déjà fait concrètement avec la «contribution de l'ILR aux partenariats migratoires Suisse-Balkans occidentaux 2007-2009».

\section{Bibliographie}

Eidgenössische Finanzkontrolle (EFK), Bundesamt für Migration, Bereich Rückkehrförderung, EFK Bericht, $n^{\circ}$ 1.6196.420.00145.02, Bern, EFK, 20. Dezember 2006.

Equipe de projet Balkans, Groupe directeur interdépartemental d'aide au retour (ILR), Stratégie Balkans 2003-2006: Programme d'aide au retour dans les Balkans à l'intention des personnes vulnérables (Bosnie-et-Herzégovine, Serbie, Monténégro et Kosovo): Rapport final 2007 (période du $1^{\text {er }}$ juillet 2003 au 31 décembre 2006), Berne, Office fédéral des migrations (ODM); Direction du développement et de la coopération (DDC); Organisation internationale pour les migrations (OIM), 2007.

Groupe directeur interdépartemental d'aide au retour (ILR), Contribution de l'ILR concernant les partenariats migratoires entre la Suisse et les Balkans occidentaux: Stratégie 2007-2009, Kosovo, Bosnieet-Herzégovine, Serbie, Berne, Office fédéral des migrations (ODM); Direction du développement et de la coopération (DDC), 2007.

Groupe directeur interdépartemental d'aide au retour (ILR), Mandat, Berne, Office fédéral des migrations (ODM); Direction du développement et de la coopération (DDC), 2005.

Sektion Rückkehrförderung BFM, Grundlagenpapier zuhanden der ILR 2008: Operationelle Instrumente der Rückkehrhilfe, Bern, Bundesamt für Migration (BFM), 2008. 